\title{
STOCHASTIC PERRON'S METHOD AND VERIFICATION WITHOUT SMOOTHNESS USING VISCOSITY COMPARISON: THE LINEAR CASE
}

\author{
ERHAN BAYRAKTAR AND MIHAI SÎRBU
}

(Communicated by Edward C. Waymire)

\begin{abstract}
We introduce a stochastic version of the classical Perron's method to construct viscosity solutions to linear parabolic equations associated to stochastic differential equations. Using this method, we construct easily two viscosity (sub- and super-) solutions that squeeze in between the expected payoff. If a comparison result holds true, then there exists a unique viscosity solution which is a martingale along the solutions of the stochastic differential equation. The unique viscosity solution is actually equal to the expected payoff. This amounts to a verification result (Itô's Lemma) for non-smooth viscosity solutions of the linear parabolic equation.
\end{abstract}

\section{INTRODUCTION}

The best way to approach a Feynman-Kac equation (or a Hamilton-JacobiBellman equation in the case of stochastic control) is to prove existence of a smooth solution and then use Itô's Lemma to relate it to the corresponding probabilistic representation. In case such a smooth solution does not exist, a large number of results in the literature consist in taking the expected payoff (value function) associated to a Markov diffusion and then checking the viscosity solution property. Such an approach essentially uses the Markov property of the diffusion. If uniqueness in law of the stochastic differential equation does not hold, then a Markov selection is needed to obtain a viscosity solution this way. If, in addition, a viscosity comparison result holds true (which is a purely analytical result), then the conclusion is that the expected payoff (value function) is actually the unique viscosity solution.

On the other hand, Ishii [2] refined the classical Perron's method to the case of viscosity solutions. This amounts to a very powerful analytical method to construct (therefore proving existence) viscosity solutions in very general frameworks. However, if one wants to compare such a viscosity solution obtained by Perron's method with the expected payoff (value function), then one still needs the viscosity property for the expected payoff (value function). In other words, the program described in the beginning still needs to be carried out.

Received by the editors April 14, 2011.

2010 Mathematics Subject Classification. Primary 60G46, 60H30; Secondary 35K65, 35K10.

Key words and phrases. Perron's method, viscosity solutions, non-smooth verification, comparison principle.

The research of the first author was supported in part by the National Science Foundation under grants DMS 0906257 and DMS 0955463.

The research of the second author was supported in part by the National Science Foundation under Grant DMS 0908441.

(C) 2012 American Mathematical Society Reverts to public domain 28 years from publication 
In this paper, we propose a stochastic alternative to Perron's method to construct viscosity solutions, namely Theorem 2.1. More precisely, we consider the infimum of stochastic super-solutions or the supremum of stochastic sub-solutions to a linear parabolic PDE. By stochastic sub-solutions and super-solutions we mean obvious generalizations of the seminal notion of stochastic solution introduced by Stroock and Varadhan [5]. To the best of our knowledge, such a technique does not exist in the literature. While this construction does not provide a stochastic solution, it does provide a (weaker) viscosity solution. The main advantage of our method is that comparison between such constructed viscosity solutions and the expected payoff (value function) becomes trivial (see Lemma 2.1), because it is imbedded in the stochastic definition. In other words, one does not need to prove any property for the expected payoff (value function) in order to compare it to the viscosity solution(s) constructed by the stochastic Perron method. Using this result, if viscosity comparison holds, then one gets that the unique viscosity solution is actually equal to the expected payoff (value function) for free. The unique viscosity solution is a martingale along any solution of the stochastic differential equation, i.e. is a stochastic solution in the sense of Stroock and Varadhan [5. This actually amounts to a verification result for non-smooth viscosity solutions, where we can use the uniqueness of viscosity solutions as a substitute for verification.

In the present paper we illustrate these ideas in the simplest framework of linear parabolic equations with terminal conditions on the whole state space (a particular version of Feynman-Kac). However, we claim that these ideas carry over to much more general frameworks. In particular, other linear cases including infinite horizons, running costs, exit times or even reflections on the boundary can be easily treated in an identical way. More interestingly, we intend to carry over these ideas to the more important case of Hamilton-Jacobi-Bellman equations associated to stochastic control and stochastic games (Isaac's equations). These more technical details are left to future work and will be presented in forthcoming papers.

\section{The Setup And Main Results}

Fix a time interval $T>0$ and for each $0 \leq s<T$ and $x \in \mathbb{R}^{d}$ consider the stochastic differential equation

$$
\left\{\begin{array}{l}
d X_{t}=b\left(t, X_{t}\right) d t+\sigma\left(t, X_{t}\right) d W_{t}, \quad s \leq t \leq T, \\
X_{s}=x .
\end{array}\right.
$$

We assume that the coefficients $b:[0, T] \times \mathbb{R}^{d} \rightarrow \mathbb{R}^{d}$ and $\sigma:[0, T] \times \mathbb{R}^{d} \rightarrow \mathbb{M}_{d, d^{\prime}}(\mathbb{R})$ are continuous. We also assume that, for each $(s, x)$, equation (1) has at least a weak non-exploding solution

$$
\left(\left(X_{t}^{s, x}\right)_{s \leq t \leq T},\left(W_{t}^{s, x}\right)_{s \leq t \leq T}, \Omega^{s, x}, \mathcal{F}^{s, x}, \mathbb{P}^{s, x},\left(\mathcal{F}_{t}^{s, x}\right)_{s \leq t \leq T}\right),
$$

where the $W^{s, x}$ is a $d^{\prime}$-dimensional Brownian motion on the stochastic basis

$$
\left(\Omega^{s, x}, \mathcal{F}^{s, x}, \mathbb{P}^{s, x},\left(\mathcal{F}_{t}^{s, x}\right)_{s \leq t \leq T}\right)
$$

and the filtration $\left(\mathcal{F}_{t}^{s, x}\right)_{s \leq t \leq T}$ satisfies the usual conditions. We denote by $\mathcal{X}^{s, x}$ the non-empty set of such weak solutions. It is well known, for example from [4, that a sufficient condition for the existence of non-exploding solutions, in addition to continuity of the coefficients, is the condition of linear growth:

$$
|b(t, x)|+|\sigma(t, x)| \leq C(1+|x|), \quad(t, x) \in[0, T] \times \mathbb{R}^{d} .
$$


We emphasize that we do not assume uniqueness in law of the weak solution.

Remark 2.1. Actually, in order to insure that $\mathcal{X}^{s, x}$ is a set in the sense of axiomatic set theory, one should restrict to weak solutions where the probability space $\Omega$ is an element of a fixed universal set $\mathcal{S}$ of possible probability spaces.

Now, for some fixed bounded and measurable function $g: \mathbb{R}^{d} \rightarrow \mathbb{R}$, we denote by

$$
v_{*}(s, x):=\inf _{\mathcal{X}^{s, x}} \mathbb{E}^{s, x}\left[g\left(X_{T}^{s, x}\right)\right] \text { and } v^{*}(s, x):=\sup _{\mathcal{X}^{s, x}} \mathbb{E}^{s, x}\left[g\left(X_{T}^{s, x}\right)\right] .
$$

We will call the functions $v_{*}$ and $v^{*}$ the lower and the upper expected payoffs (value functions). It is obvious that

$$
v_{*} \leq v^{*}
$$

and the two functions coincide if the stochastic differential equation (1) has a unique in law weak solution.

Remark 2.2. At this stage, we cannot even conclude that $v_{*}$ and $v^{*}$ are measurable.

We believe that the expected payoffs (value functions) $v_{*}$ and $v^{*}$ will be associated to the linear PDE

$$
\left\{\begin{array}{l}
-u_{t}-L_{t} u=0 \\
u(T, x)=g(x)
\end{array}\right.
$$

where the time-dependent operator $L_{t}$ is defined by

$$
\left(L_{t} u\right)(x)=\langle b(t, x), \nabla u(t, x)\rangle+\frac{1}{2} \operatorname{Tr}\left(\sigma(t, x) \sigma^{T}(t, x) u_{x x}(t, x)\right), \quad 0 \leq t<T, x \in \mathbb{R}^{d} .
$$

2.1. Stochastic Perron's method. Let $g: \mathbb{R}^{d} \rightarrow \mathbb{R}$ be measurable and bounded. As mentioned in the Introduction, we now introduce the sets of stochastic supersolutions and sub-solutions of the parabolic PDE (2) in the spirit of [5].

Definition 2.1. The set of stochastic super-solutions of the parabolic PDE (2), denoted by $\mathcal{U}^{+}$, is the set of functions $u:[0, T] \times \mathbb{R}^{d} \rightarrow \mathbb{R}$ which have the following properties:

(1) They are upper semicontinuous (USC) and bounded on $[0, T] \times \mathbb{R}^{d}$. In addition, they satisfy the terminal condition $u(T, x) \geq g(x)$ for all $x \in \mathbb{R}^{d}$.

(2) For each $(s, x) \in[0, T] \times \mathbb{R}^{d}$ and each weak solution

$$
\left(\left(X_{t}^{s, x}\right)_{s \leq t \leq T},\left(W_{t}^{s, x}\right)_{s \leq t \leq T}, \Omega^{s, x}, \mathcal{F}^{s, x}, \mathbb{P}^{s, x},\left(\mathcal{F}_{t}^{s, x}\right)_{s \leq t \leq T}\right) \in \mathcal{X}^{s, x},
$$

the process $\left(u\left(t, X_{t}^{s, x}\right)\right)_{s \leq t \leq T}$ is a supermartingale on $\left(\Omega^{s, x}, \mathbb{P}^{s, x}\right)$ with respect to the filtration $\left(\mathcal{F}_{t}^{s, \bar{x}}\right)_{s \leq t \leq T}$.

Definition 2.2. The set of stochastic sub-solutions of the parabolic PDE (2), denoted by $\mathcal{U}^{-}$, is the set of functions $u:[0, T] \times \mathbb{R}^{d} \rightarrow \mathbb{R}$ which have the following properties:

(1) They are lower semicontinuous (LSC) and bounded on $[0, T] \times \mathbb{R}^{d}$. In addition, they satisfy the terminal condition $u(T, x) \leq g(x)$ for all $x \in \mathbb{R}^{d}$.

(2) For each $(s, x) \in[0, T] \times \mathbb{R}^{d}$ and each weak solution

$$
\left(\left(X_{t}^{s, x}\right)_{s \leq t \leq T},\left(W_{t}^{s, x}\right)_{s \leq t \leq T}, \Omega^{s, x}, \mathcal{F}^{s, x}, \mathbb{P}^{s, x},\left(\mathcal{F}_{t}^{s, x}\right)_{s \leq t \leq T}\right) \in \mathcal{X}^{s, x},
$$

the process $\left(u\left(t, X_{t}^{s, x}\right)\right)_{s \leq t \leq T}$ is a submartingale on $\left(\Omega^{s, x}, \mathbb{P}^{s, x}\right)$ with respect to the filtration $\left(\mathcal{F}_{t}^{s, x}\right)_{s \leq t \leq T}$. 
Remark 2.3. In the Definitions 2.1, 2.2 of $\mathcal{U}^{+}, \mathcal{U}^{-}$we do not assume that the processes $\left(u\left(t, X_{t}^{s, x}\right)\right)_{s \leq t \leq T}$ have (RC) right-continuous paths. For this reason, care must be taken when one tries to apply the Optional Sampling Theorem in the form of "a stopped martingale is a martingale". More precisely, such a theorem holds only with respect to discrete-valued stopping times.

Remark 2.4. Since $g$ is assumed bounded, the sets $\mathcal{U}^{-}$and $\mathcal{U}^{+}$are easily seen to be non-empty. More precisely any constant function $u(t, x) \equiv k$ which is an upper bound to $g(g \leq k)$ is in $\mathcal{U}^{+}$and any constant function $u(t, x) \equiv k$ which is a lower bound to $g(k \leq g)$ is in $\mathcal{U}^{-}$. If one wants to account for a larger class of functions $g$ than bounded, then the definitions of $\mathcal{U}^{-}$and $\mathcal{U}^{+}$should be changed appropriately, and an assumption on the non-emptiness of $\mathcal{U}^{-}$and $\mathcal{U}^{+}$should be made.

Using the properties of sub (super)martingales as well as the definition of $v_{*}$ and $v^{*}$, we easily obtain the following result.

Lemma 2.1. For each $u \in \mathcal{U}^{-}$and each $w \in \mathcal{U}^{+}$we have $u \leq v_{*} \leq v^{*} \leq w$.

Using Remark 2.4 and Lemma 2.1, we can define

$$
v^{-}:=\sup _{u \in \mathcal{U}^{-}} u \leq v_{*} \leq v^{*} \leq v^{+}:=\inf _{w \in \mathcal{U}^{+}} w .
$$

Lemma 2.2. We have $v^{-} \in \mathcal{U}^{-}, v^{+} \in \mathcal{U}^{+}$.

Proof. It is well known that an infimum of upper semicontinous functions is upper semicontinuous. While we cannot conclude directly that the pointwise infimum of supermartingales is a supermartingale (because the set of supermartingales may be uncountable, and the use of an essential infimum would be needed), we can appeal to Proposition 4.1 in the Appendix and conclude that $v^{+}$is actually the pointwise infimum of a countable set of functions $w_{n} \in \mathcal{U}^{+}, n=1,2 \ldots$ Now, the pointwise infimum of a countable set of supermartingales is, indeed, a supermartingale. The terminal condition for $v^{+}$is satisfied and the boundedness follows easily since $g$ is bounded, so $v^{*}$ is bounded, and using Remark 2.4 and Lemma 2.1 we have

$$
v^{*} \leq v^{+} \leq \sup _{x \in \mathbb{R}^{d}} g(x) .
$$

Therefore $v^{+} \in U^{+}$. The other part is identical.

Remark 2.5. Using Lemma 2.2, one could easily show that $v^{+}$is a viscosity supersolution of (2) (i.e. satisfies (8) below in the viscosity sense) and $v^{-}$is a viscosity sub-solution of (2) (i.e. satisfies (7) below in the viscosity sense). However, while true, this is not of much interest.

The following is the main technical result of the present paper:

Theorem 2.1 (Stochastic Perron's Method). If $g$ is bounded and $L S C$, then $v^{-}$is a bounded and LSC viscosity super-solution of

$$
\left\{\begin{array}{l}
-u_{t}-L_{t} u \geq 0 \\
u(T, x) \geq g(x)
\end{array}\right.
$$

If $g$ is bounded and USC, then $v^{+}$is a bounded and USC viscosity sub-solution of

$$
\left\{\begin{array}{l}
-u_{t}-L_{t} u \leq 0 \\
u(T, x) \leq g(x)
\end{array}\right.
$$


Remark 2.6. We have $v^{-}(T, x) \leq g(x)$ and $v^{+}(T, x) \geq g(x)$ by construction. Therefore, the terminal conditions in (3) and (4) can be replaced by equalities.

Remark 2.7. We would like to point out that the semicontinuous solutions obtained by Perron's method have the correct semicontinuity needed for such a definition. We refer the reader to [1] for an introduction to (semicontinuous) viscosity subsolutions and super-solutions of second-order equations.

Proof. We will only prove that $v^{+}$is a sub-solution of (4): the other part is symmetric.

Step 1. The interior sub-solution property. Note that we already know that $v^{+}$is bounded and upper semicontinuous. Let

$$
\varphi:[0, T] \times \mathbb{R}^{d} \rightarrow \mathbb{R}
$$

be a $C^{1,2}$-test function and assume that $v^{+}-\varphi$ attains a strict local maximum (an assumption which is not restrictive) equal to zero at some interior point $\left(t_{0}, x_{0}\right) \in$ $(0, T) \times \mathbb{R}^{d}$. Assume that $v^{+}$does not satisfy the viscosity sub-solution property, and therefore

$$
-\varphi_{t}\left(t_{0}, x_{0}\right)-\mathcal{L}_{t} \varphi\left(t_{0}, x_{0}\right)>0 .
$$

Since the coefficients of the SDE are continuous, we conclude that there exists a small enough ball $B\left(t_{0}, x_{0}, \varepsilon\right)$ such that

$$
-\varphi_{t}-\mathcal{L}_{t} \varphi>0 \text { on } \overline{B\left(t_{0}, x_{0}, \varepsilon\right)}
$$

and

$$
\varphi>v^{+} \text {on } \overline{B\left(t_{0}, x_{0}, \varepsilon\right)}-\left(t_{0}, x_{0}\right) .
$$

Since $v^{+}-\varphi$ is upper semicontinuous and $\overline{B\left(t_{0}, x_{0}, \varepsilon\right)}-B\left(t_{0}, x_{0}, \varepsilon / 2\right)$ is compact, this means that there exists a $\delta>0$ such that

$$
\varphi-\delta \geq v^{+} \text {on } \overline{B\left(t_{0}, x_{0}, \varepsilon\right)}-B\left(t_{0}, x_{0}, \varepsilon / 2\right) .
$$

Now, if we choose $0<\eta<\delta$ we have that the function

$$
\varphi_{\eta}=\varphi-\eta
$$

satisfies the properties

$$
\begin{gathered}
-\varphi_{t}^{\eta}-\mathcal{L}_{t} \varphi^{\eta}>0 \text { on } \overline{B\left(t_{0}, x_{0}, \varepsilon\right)}, \\
\varphi^{\eta}>v^{+} \text {on } \overline{B\left(t_{0}, x_{0}, \varepsilon\right)}-B\left(t_{0}, x_{0}, \varepsilon / 2\right),
\end{gathered}
$$

and

$$
\varphi^{\eta}\left(t_{0}, x_{0}\right)=v^{+}\left(t_{0}, x_{0}\right)-\eta
$$

Now, we define the new function

$$
v^{\eta}=\left\{\begin{array}{l}
v^{+} \wedge \varphi^{\eta} \text { on } \overline{B\left(t_{0}, x_{0}, \varepsilon\right)}, \\
v^{+} \text {outside } \overline{B\left(t_{0}, x_{0}, \varepsilon\right)} .
\end{array}\right.
$$

We clearly have that $v^{\eta}$ is upper semicontinous and $v^{\eta}\left(t_{0}, x_{0}\right)=\varphi^{\eta}\left(t_{0}, x_{0}\right)<$ $v^{+}\left(t_{0}, x_{0}\right)$. Also, $v^{\eta}$ satisfies the terminal condition (since $\varepsilon$ can be chosen so that $T>t_{0}+\varepsilon$ and $v^{+}$satisfies the terminal condition). It only remains to show that $v^{\eta} \in \mathcal{U}^{+}$to obtain a contradiction. For the analytical Perron method on viscosity solutions, the proof would now be finished, since the viscosity solution property is local and the minimum of two super-solutions is a super-solution. In our case, 
the supermartingale property defining $\mathcal{U}^{+}$is global, so we need to localize it using stopping times. Particular care has to be taken since the paths may not be right-continuous, so localization in general may fail, as pointed out in Remark 2.3.

Fix $(s, x)$ and $\left(\left(X_{t}^{s, x}\right)_{s \leq t \leq T},\left(W_{t}^{s, x}\right)_{s \leq t \leq T}, \Omega^{s, x}, \mathcal{F}^{s, x}, \mathbb{P}^{s, x},\left(\mathcal{F}_{t}^{s, x}\right)_{s \leq t \leq T}\right) \in \mathcal{X}^{s, x}$. We need to show that the process $\left(v^{\eta}\left(t, X_{t}^{s, x}\right)\right)_{s \leq t \leq T}$ is a supermartingale on $\left(\Omega^{s, x}, \mathbb{P}^{s, x}\right)$ with respect to the filtration $\left(\mathcal{F}_{t}^{s, x}\right)_{s \leq t \leq T}$. We first do the proof under the additional assumption that the process $\left(v^{+}\left(t, X_{t}^{s, x}\right)\right)_{s \leq t \leq T}$ does have RC paths.

Under this assumption, the process $\left(v^{\eta}\left(t, X_{t}^{s, x}\right)\right)_{s \leq t \leq T}$ is a supermartingale locally in the region $[s, T] \times \mathbb{R}^{d}-B\left(t_{0}, x_{0}, \varepsilon / 2\right)$ because it coincides there with the process $\left(v^{+}\left(t, X_{t}^{s, x}\right)\right)_{s \leq t \leq T}$ which is an RC supermartingale, so it can be localized. In addition, in the region $B\left(t_{0}, x_{0}, \varepsilon\right)$ the process $\left(v^{\eta}\left(t, X_{t}^{s, x}\right)\right)_{s \leq t \leq T}$ is the minimum between two local supermartingales, therefore a local supermartingale. (It is clear that the process $\left(\varphi^{\eta}\left(t, X_{t}^{s, x}\right)\right)_{s \leq t \leq T}$ is a local supermartingale over $B\left(t_{0}, x_{0}, \varepsilon\right)$ by Itô's formula.) Since the two regions $[s, T] \times \mathbb{R}^{d}-B\left(t_{0}, x_{0}, \varepsilon / 2\right)$ and $B\left(t_{0}, x_{0}, \varepsilon\right)$ actually overlap over an open region, then we can conclude that the process $\left(v^{\eta}\left(t, X_{t}^{s, x}\right)\right)_{s \leq t \leq T}$ is indeed a supermartingale. In order to make this argument, one needs to choose a double sequence of stopping times reminiscent of the optimal strategy in switching control problems. More precisely, the double sequence is chosen as the times exiting from $B\left(t_{0}, x_{0}, \varepsilon\right)$ and the sequel times entering $B\left(t_{0}, x_{0}, \varepsilon / 2\right)$. The choice depends on where the process actually is at the initial time $s$.

In general, i.e., if the process $\left(v^{+}\left(t, X_{t}^{s, x}\right)\right)_{s \leq t \leq T}$ does not have RC paths, then we can work with its right-continuous limit over rational times to reduce it to the case above. More precisely, fix $0 \leq s \leq r \leq t \leq T$ and $x \in \mathbb{R}^{d}$. We want to prove the supermartingale property for the process $\left(Y_{u}\right)_{s \leq u \leq T}:=\left(v^{\eta}\left(u, X_{u}^{s, x}\right)\right)_{s \leq u \leq T}$ between the times $r$ and $t$, which means we want to show that

$$
Y_{r} \geq \mathbb{E}^{s, x}\left[Y_{t} \mid \mathcal{F}_{r}^{s, x}\right]
$$

First, we make the notation $Z_{u}:=v^{+}\left(u, X_{u}^{s, x}\right)$ for $r \leq u \leq t$ and we stop it at time $t$, i.e. $Z_{u}:=v^{+}\left(t, X_{t}^{s, x}\right)$ for $t \leq u \leq T$. The process $\left(Z_{u}\right)_{r \leq u \leq T}$ is a supermartingale, but may not be RC, as discussed. We can use Proposition 3.14, page 16, in Karatzas and Shreve [3] to define the RC supermartingale

$$
Z_{u}^{+}(\omega):=\lim _{q \rightarrow u, q>u, q \in \mathbb{Q}} Z_{q}(\omega), \quad \omega \in \Omega^{*}, r \leq u \leq T,
$$

and

$$
Z^{+}=0, \omega \notin \Omega^{*},
$$

where $\mathbb{P}^{s, x}\left[\Omega^{*}\right]=1$. We would like to emphasize that $Z^{+}$is, indeed, an RC supermartingale with respect to the original filtration since the filtration is assumed to satisfy the usual conditions. Since the function $v^{+}$is USC, and the process is constant after $t$ we can conclude (taking pathwise limits) that

$$
Z_{r} \geq Z_{r}^{+}, \quad Z_{t}=Z_{t}^{+} .
$$

We recall that in the open region $B\left(t_{0}, x_{0}, \varepsilon\right)-\overline{B\left(t_{0}, x_{0}, \varepsilon / 2\right)}$ we have $v^{+}<\varphi-\delta$. Therefore, if we take right limits inside this region and use the fact that $\varphi$ is continuous, then we get

$$
Z_{u}^{+}<\varphi^{\eta}\left(u, X_{u}^{s, x}\right) \text { if }\left(u, X_{u}^{s, x}\right) \in B\left(t_{0}, x_{0}, \varepsilon\right)-\overline{B\left(t_{0}, x_{0}, \varepsilon / 2\right)} .
$$


Now, we can define the process

$$
Y_{u}^{+}:=\left\{\begin{array}{l}
Z_{u}^{+}, \quad\left(u, X_{u}^{s, x}\right) \notin \overline{B\left(t_{0}, x_{0}, \varepsilon / 2\right)} \\
Z_{u}^{+} \wedge \varphi^{\eta}\left(u, X_{u}^{s, x}\right), \quad\left(u, X_{u}^{s, x}\right) \in B\left(t_{0}, x_{0}, \varepsilon\right) .
\end{array}\right.
$$

We note that we have

$$
Y_{r} \geq Y_{r}^{+}, \quad Y_{t}=Y_{t}^{+} .
$$

Now, for the process $Y^{+}$, we can apply the previous argument, since $Z^{+}$has RC paths, to conclude it is a supermartingale. In particular, we have that

$$
Y_{r} \geq Y_{r}^{+} \geq \mathbb{E}^{s, x}\left[Y_{t}^{+} \mid \mathcal{F}_{r}^{s, x}\right]=\mathbb{E}^{s, x}\left[Y_{t} \mid \mathcal{F}_{r}^{s, x}\right]
$$

Step 2. The terminal condition. Assume that, for some $x_{0} \in \mathbb{R}^{d}$ we have $v^{+}\left(T, x_{0}\right)>g\left(x_{0}\right)$. We want to use this information in a similar way to Step 1 to construct a contradiction. Since $g$ is USC on $\mathbb{R}^{d}$, there exists an $\varepsilon>0$ such that

$$
g(x) \leq v^{+}\left(T, x_{0}\right)-\varepsilon, \quad\left|x-x_{0}\right| \leq \varepsilon .
$$

We now use the fact that $v^{+}$is USC to conclude it is bounded above on the compact set

$$
\left(\overline{B\left(T, x_{0}, \varepsilon\right)}-B\left(T, x_{0}, \varepsilon / 2\right)\right) \cap\left([0, T] \times \mathbb{R}^{d}\right) .
$$

This was anyway clear, since actually $v^{+}$is globally bounded, but the argument above shows the proof works in even more general cases. Now, we choose $\eta>0$ small enough so that

$$
v^{+}\left(T, x_{0}\right)+\frac{\varepsilon^{2}}{4 \eta} \geq \varepsilon+\sup _{(t, x) \in\left(\overline{B\left(T, x_{0}, \varepsilon\right)}-B\left(T, x_{0}, \varepsilon / 2\right)\right) \cap\left([0, T] \times \mathbb{R}^{d}\right)} v^{+}(t, x) .
$$

We now define, for $k>0$ the following function:

$$
\varphi^{\eta, \varepsilon, k}(t, x)=v^{+}\left(T, x_{0}\right)+\frac{\left|x-x_{0}\right|^{2}}{\eta}+k(T-t) .
$$

For $k$ large enough we have that

$$
-\varphi_{t}^{\varepsilon, \eta, k}-\mathcal{L}_{t} \varphi^{\varepsilon, \eta, k}>0, \quad \text { on } \overline{B\left(T, x_{0}, \varepsilon\right)} .
$$

In addition, using (6) we have that, for $k \geq \varepsilon / 2 \eta$,

$$
\varphi^{\varepsilon, \eta, k} \geq \varepsilon+v^{+} \text {, on }\left(\overline{B\left(T, x_{0}, \varepsilon\right)}-B\left(T, x_{0}, \varepsilon / 2\right)\right) \cap\left([0, T] \times \mathbb{R}^{d}\right) .
$$

Also, $\varphi^{\varepsilon, \eta, k}(T, x) \geq v^{+}\left(T, x_{0}\right) \geq g(x)+\varepsilon$ for $\left|x-x_{0}\right| \leq \varepsilon$. Now, we can choose $\delta<\varepsilon$ and define as in the proof of Step 1,

$$
v^{\varepsilon, \eta, k, \delta}=\left\{\begin{array}{l}
v^{+} \wedge\left(\varphi^{\varepsilon, \eta, k}-\delta\right) \text { on } \overline{B\left(T, x_{0}, \varepsilon\right)} \\
v^{+} \text {outside } \overline{B\left(T, x_{0}, \varepsilon\right)}
\end{array}\right.
$$

We can now prove, using the same switching principle and RC modification argument as in Step 1, that $v^{\varepsilon, \eta, k, \delta} \in \mathcal{U}^{+}$, but $v^{\varepsilon, \eta, k, \delta}\left(T, x_{0}\right)=v^{+}\left(T, x_{0}\right)-\delta$, leading to a contradiction. 


\subsection{Verification by comparison.}

Definition 2.3. We say that the viscosity comparison principle holds for the equation (2) with respect to the time horizon $T$ and the final condition $g$ or that condition $C P(T, g)$ is satisfied if, whenever we have a bounded, upper semicontinuous (USC) subsolution $u$ of

$$
\left\{\begin{array}{l}
-u_{t}-L_{t} u \leq 0 \\
u(T, x) \leq g(x)
\end{array}\right.
$$

and a bounded lower semicontinous super-solution $v$ of

$$
\left\{\begin{array}{l}
-u_{t}-L_{t} u \geq 0 \\
u(T, x) \geq g(x)
\end{array}\right.
$$

then $u \leq v$.

The next theorem is an easy consequence of our main result, Theorem 2.1. However, it amounts to a verification result for non-smooth viscosity solutions of (2), so we consider it to be the other main result of the present paper.

Theorem 2.2. Let $g$ be bounded and continuous. Assume also that the comparison principle $C P(T, g)$ is satisfied. Then there exists a unique bounded and continuous viscosity solution $v$ to (2) which equals both the lower and the upper payoffs (value functions), which means

$$
v_{*}=v=v^{*} .
$$

In addition, for each $(s, x) \in[0, T] \times \mathbb{R}^{d}$, and each weak solution

$$
\left(\left(X_{t}^{s, x}\right)_{s \leq t \leq T},\left(W_{t}^{s, x}\right)_{s \leq t \leq T}, \Omega^{s, x}, \mathcal{F}^{s, x}, \mathbb{P}^{s, x},\left(\mathcal{F}_{t}^{s, x}\right)_{s \leq t \leq T}\right) \in \mathcal{X}^{s, x},
$$

the process $\left(v\left(t, X^{s, x}\right)\right)_{s \leq t \leq T}$ is a martingale on $\left(\Omega^{s, x}, \mathbb{P}^{s, x}\right)$ with respect to the filtration $\left(\mathcal{F}_{t}^{s, x}\right)_{s \leq t \leq T}$.

Proof. The proof is immediate in light of Definition 2.3. Lemma 2.5 and Theorem 2.1

Remark 2.8. The martingale property for $\left(v\left(t, X^{s, x}\right)\right)_{s \leq t \leq T}$ is proved without using the Markov property of the weak solution (which is not even assumed). However, if $C P(T, g)$ is satisfied for any $T$ and any bounded (test function) $g$, then we obtain that, for each $(s, x)$ and each $T$, the law of $X_{T}^{s, x}$ is uniquely determined. Following Theorem 6.2.3 in [4] or Proposition 4.27, page 326, in [3], uniqueness of marginals implies uniqueness in law of the weak solution. Now, uniqueness in law for any $(s, x)$ does imply the Markov property for the weak solution of the SDE (11) (the Markov property holds with respect to the natural raw filtration though). We refer the reader to Theorem 6.2.2 in [4] or Theorem 4.20, page 322, in [3] for the last-mentioned result.

Remark 2.9. The whole paper can be rewritten by selecting, for each $(s, x)$, only one weak solution $X^{s, x}$ instead of using the set of weak solutions $\mathcal{X}^{s, x}$. Such a selection uses the axiom of choice and does not need to be a Markov selection. Once the selection $X^{s, x}$ is chosen, the sets of stochastic super-solutions and subsolutions in Definitions 2.1 and 2.2 have to be redefined accordingly, and there is only one payoff (value function) $v$ replacing $v_{*}$ and $v^{*}$. 


\section{Conclusions}

We designed a stochastic counterpart to Perron's method which produces two viscosity solutions of the Feynman-Kac equation. The two solutions squeeze in between the expected payoff, and this comparison is a trivial consequence of the probabilistic definition. If, in addition, a viscosity comparison result holds, then we do have a unique viscosity solution, which is a martingale along the solutions of the stochastic differential equation and is equal to the expected payoff. In this case, we therefore have a full verification result without smoothness of the viscosity solution.

While the Perron method we describe here is reminiscent of the characterization of the value function in optimal stopping problems as the least excessive function, we would like to point out that here, unlike in optimal stopping, we avoid proving that the value function is "excessive". This is actually the point of verification by comparison to avoid working with the value function.

One could try to prove directly, avoiding viscosity altogether, that $v^{+}$and $v^{-}$ along solutions of the SDE are martingales; i.e., they are stochastic solutions in the sense of Stroock and Varadhan [5]. However, this is actually not possible: $v^{+}$and $v^{-}$are stochastic solutions only when they coincide, since the stochastic solutions are unique by definition. We can additionally justify that, in general, $v^{+}$ and $v^{-}$are viscosity solutions but may not be stochastic solutions by observing that the stochastic solution property is much stronger than viscosity. Fortunately, in a large number of situations, the viscosity property is still strong enough to prove uniqueness. In this case, viscosity and the stochastic solution property are equivalent.

\section{Appendix: Countable selection to achieve the inf/sup OF A CLASS OF SEMICONTINUOUS FUNCTIONS}

The main purpose of the Appendix is to prove a countable selection argument needed in the proof of Lemma 2.1. Let $(M, d)$ be a metric space and consider a class $\mathcal{G}$ of functions $f: M \rightarrow \overline{\mathbb{R}}$. The first result is

Lemma 4.1. Let $g: M \rightarrow \overline{\mathbb{R}}$. Then, the following conditions are equivalent:

(1) $g(x)=\inf _{f \in \mathcal{G}} f(x)$, for each $x \in M$,

(2) $\{x \in M \mid g(x)<q\}=\bigcup_{f \in \mathcal{G}}\{x \in M \mid f(x)<q\}$, for each $q \in \mathbb{Q}$.

Proposition 4.1. Assume that $(M, d)$ is a separable metric space (or, less, a topological space with a countable base). Assume also that each function in the class $\mathcal{G}$ is upper semicontinuous (USC). Then, there exists a countable subclass of functions $\mathcal{H} \subset \mathcal{G}$ such that

$$
f_{*}(x):=\inf _{f \in \mathcal{G}} f(x)=\inf _{f \in \mathcal{H}} f(x), \text { for each } x \in M .
$$

Proof. Fix a $q \in \mathbb{Q}$. According to Lemma 4.1, the open set $\left\{x \in M \mid f_{*}(x)<q\right\}$ admits an open cover as

$$
\left\{x \in M \mid f_{*}(x)<q\right\}=\bigcup_{f \in \mathcal{G}}\{x \in M \mid f(x)<q\} .
$$


Since the space $(M, d)$ is separable, so it admits a countable basis, one can select a countable open subcover. More precisely, there exists a countable $\mathcal{G}_{q} \subset \mathcal{G}$ such that

$$
\left\{x \in M \mid f_{*}(x)<q\right\}=\bigcup_{f \in \mathcal{G}_{q}}\{x \in M \mid f(x)<q\} .
$$

Now, we define the countable class

$$
\mathcal{H}:=\bigcup_{q \in \mathbb{Q}} \mathcal{G}_{q}
$$

We have, for each $q \in \mathbb{Q}$, that

$$
\begin{aligned}
\left\{x \in M \mid f_{*}(x)<q\right\} & =\bigcup_{f \in \mathcal{G}_{q}}\{x \in M \mid f(x)<q\} \subset \bigcup_{f \in \mathcal{H}}\{x \in M \mid f(x)<q\} \\
& \subset\left\{x \in M \mid f_{*}(x)<q\right\} .
\end{aligned}
$$

According to Lemma 4.1 we then have that

$$
f_{*}(x)=\inf _{f \in \mathcal{H}} f(x), \quad \text { for each } x \in M .
$$

Remark 4.1. We would like to point out that in the argument of selecting a countable open subcover, the axiom of choice is used.

\section{ACKNOWLEDGEMENTS}

The authors would like to thank Gerard Brunick and Gordan Žitković for their comments. Special thanks go to Ioannis Karatzas for his suggestions that led to an improved final version.

\section{REFERENCES}

1. M. Crandall, H. Ishii, and P.-L. Lions, User's guide to viscosity solutions of second-order partial differential equations, Bull. Amer. Math. Soc. (N.S.) 27 (1992), 1-67. MR1118699 (92j:35050)

2. H. Ishii, Perron's method for Hamilton-Jacobi equations, Duke Mathematical Journal 55 (1987), no. 2, 369-384. MR894587 (89a:35053)

3. I. Karatzas and S. Shreve, Brownian motion and stochastic calculus, Springer, New York, 1988. MR.917065(89c:60096)

4. D. W. Stroock and S. R. S. Varadhan, Multidimensional diffusion processes, Classics in Mathematics, Springer-Verlag, Berlin, 2006, reprint of the 1997 edition. MR2190038 (2006f:60005)

5. D. W. Stroock and S. R. S. Varadhan, On degenerate elliptic-parabolic operators of second order and their associated diffusions, Communications on Pure and Applied Mathematics 25 (1972), 651-713. MR0387812(52:8651)

Department of Mathematics, University of Michigan, 530 Church Street, Ann Arbor, Michigan 48109

E-mail address: erhan@umich.edu

Department of Mathematics, University of Texas at Austin, 1 University Station C1200, Austin, Texas 78712

E-mail address: sirbu@math.utexas.edu. 